\title{
Sharing the Small Cells for Energy Efficient Networking: How much does it cost?
}

\author{
Alexandra Bousia ${ }^{\triangleright}$, Elli Kartsakli`, Angelos Antonopoulos ${ }^{\dagger}$, Luis Alonso ${ }^{\diamond}$, Christos Verikoukis ${ }^{\dagger}$ \\ $\diamond$ Signal Theory and Communications Dept., Technical University of Catalonia, Barcelona, Spain \\ $\dagger$ Telecommunications Technological Center of Catalonia, Barcelona, Spain \\ Email: \{alexandra.bousia, ellik, luisg\}@tsc.upc.edu, \{aantonopoulos, cveri\}@cttc.es
}

\begin{abstract}
The explosive data traffic demands stress the need for additional capacity in cellular systems, which can be achieved through the exploitation of Small Cells (SCs). However, the deployment of SCs implies higher energy consumption in the network, something that translates into higher financial cost for the operators. To that end, the existence of a third party that provides a common SC infrastructure for the operators has been introduced as an appealing "green" solution, raising though important issues with regard to the cost sharing for the operators. In this paper, we effectively address this issue by proposing an accurate cost model for the SCs and employing different stateof-the-art techniques to share this cost. In addition, taking into account the impact of the traffic pattern on this cost, we propose a novel hybrid cost sharing policy that provides a fair outcome for the operators. Our results highlight the potential energy efficiency gains in the network along with different ways of sharing the cost of the deployed SCs.
\end{abstract}

Keywords-Infrastructure Sharing, Cost Sharing Policies, Energy Efficiency, Green Networking, Heterogeneous Networks

\section{INTRODUCTION}

Data traffic is facing an explosive growth during the last decade and it is expected to increase up to 11.2 exabytes per month by 2017 [1]. Heterogeneous Networks (HetNets), which consist of one tier of Small Cells (SCs) underlaid in traditional macro Base Stations (BSs), constitute the new trend of next generation networks for traffic offloading [3]. However, the deployment of additional infrastructure implies higher Capital and Operational Expenditures (CapEx and OpEx, respectively) for the telecommunication operators, raising at the same time the energy consumption in the whole network. To that end, the concept of an entity, known as third party, that provides the SCs to the operators has been recently introduced in the literature [2]. Therefore, leasing the infrastructure by the third party, the operators can potentially reduce their financial costs and the third party increases its income, something that results in a win-win situation.

Apart from the economic gains, the existence of an independent entity provides the opportunity for sharing the SCs resources among the operators in order to enhance the energy efficiency in the network. The concept of infrastructure sharing, which embraces the strategies and the rules that define the ways in which the resources are shared, has been recently motivated by the operators' will to provide "green" and cost effective services and solutions to the end users. Currently, over $65 \%$ of European operators are sharing their networks [4], while the regulatory constraints of infrastructure sharing are examined in [5], where the authors present the benefits of cooperation among operators. In the same context, our previous work [6] was focused on game theoretic techniques for switching off the macro BSs through infrastructure sharing between different operators.

Even though the potential gains from infrastructure sharing can be easily envisioned, there are still many challenges to overcome in order to achieve a viable business model appealing to the operators [7]. The problem becomes more intense in HetNets, where the existence of different tiers along with additional stakeholders (e.g., third party) further complicates the sharing conditions. Market practices for third party-owned SC offloading in multi-operator environments are discussed in [8], but only high level solutions are given. In [9], the network sharing problem between a single operator and multiple third party Access Points (APs) is formulated as an auction, however the leasing price is considered given. Game theoretic tools are also employed in [10] and [11] to provide pricing policies for single and multi-operator environments, respectively, when multiple third party APs are available. Nevertheless, none of these works includes a specific cost analysis. Hence, an open key question is how to divide the cost of a shared network infrastructure among the involved operators and third parties, given the heterogeneous requirements of each operator, especially in terms of traffic. Different approaches for sharing the backbone cost of wired networks, long with their discrepanceis are presented in [12] and [13]. However, the cost models are not analytically described.

In this paper, we focus on a scenario where two operators, instead of deploying their own SC infrastructure, lease the capacity of a SC network owned by a third party. We show that there are different strategies to share the SC cost among the operators, depending on the volume and the daily patterns of their traffic. The contribution of this work lies on the following points:

1) We provide an explicit model for the expenditures of the SC network, taking into account both the CapEx and the OpEx.

2) We study the outcome that different cost sharing policies provide with regard to the estimated SC cost. We identify the discrepancies of these policies, highlighting their particular traits.

3) We propose a novel cost sharing policy, called Hybrid-Sharing (HS), that combines different characteristics of already existing policies. Our proposed policy considers various aspects of the traffic patterns (i.e., traffic volume and peak times) for the cost 
sharing estimation, being less complex compared to state-of-the-art approaches.

The remainder of the paper is organized as follows. The system model along with the considered traffic patterns are described in Section II. Section III presents the models for the analytical calculation of the financial cost and the energy consumption of the SCs. In Section IV, we present four potential cost sharing policies and we introduce our proposed hybrid policy, defining the discrepancy metric between the different policies. The assessment and the discrepancies of all policies are provided in Section V. Finally, Section VI concludes the paper.

\section{SySTEM MODEL}

In this section, we present the network model of our work, along with the different traffic patterns that will be studied. We consider a set of two operators, denoted by $I=\{A, B\}$, that lease the available resources of a SC owned by a third party ${ }^{1}$. The users of each operator are uniformly distributed within the coverage area of the SC, as shown in Fig. 1.

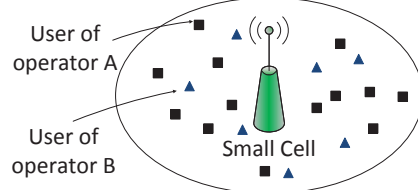

Fig. 1: Example of network layout

To quantify the cost of the SC and assess the proposed cost sharing policies, we consider different cases for the traffic patterns of the operators during the day, as it is illustrated in Fig. 2. In particular, different pricing schemes can move the traffic peaks in different time periods during the day, while the traffic distribution is affected by the specific location of the SC. For example, in urban areas, the traffic follows the typical pseudo-sinusoidal teletraffic pattern, while in places such as malls or university campuses, the traffic can be extremely bursty during specific hours within a day. Accordingly, we can distinguish the following cases for the two operators:

- $\quad$ Case I (Fig. 2a): Typical traffic - Peaks at the same time

- $\quad$ Case II (Fig. 2b): Typical traffic - Peaks at different time

- $\quad$ Case III (Fig. 2c): Bursty traffic - Peaks at the same time

- $\quad$ Case IV (Fig. 2d): Bursty traffic - Peaks at different time

From the traffic patters of Fig. 2 we can derive the total traffic $\Lambda_{i}(T)$ of operator $i \in\{A, B\}$ over a specific time interval $t \in[0, T]$ as:

$$
\Lambda_{i}(T)=\int_{0}^{T} \lambda_{i}(t) \mathrm{d} t
$$

\footnotetext{
${ }^{1}$ Even though we only consider two operators in this work, the proposed cost sharing models can also be applied in large scale networks where more than two operators are present.
}

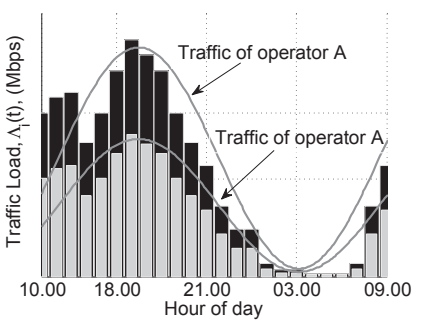

(a)

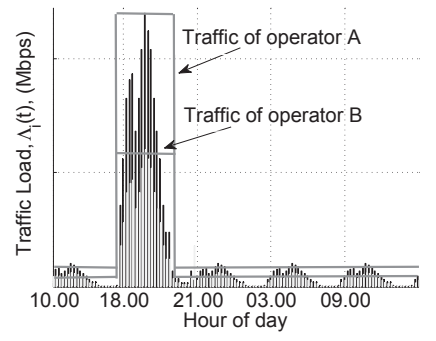

(c)

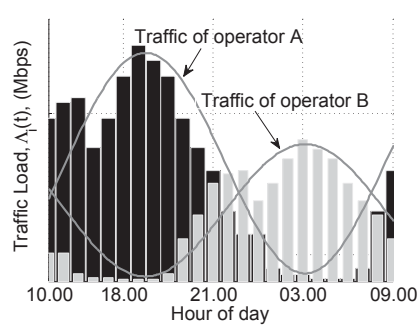

(b)

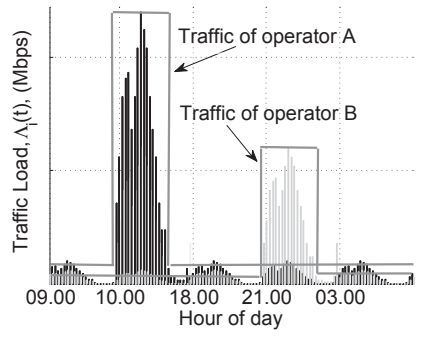

(d)
Fig. 2: Daily traffic variations for two operators: (a) Typical traffic - Peaks at the same time, (b) Typical traffic - Peaks at different time, (c) Bursty traffic - Peaks at the same time, (d) Bursty traffic - Peaks at different time

where $\lambda_{i}(t)$ is the operator's traffic load at time $t$.

We also define the parameter a to express the relationship between the total traffic loads of the two operators $A$ and $B$ :

$$
\Lambda_{B}(T)=\mathrm{a} \cdot \Lambda_{A}(T), \mathrm{a} \in \mathbb{R}^{+} .
$$

When the two operators share the SC, their aggregated traffic must not exceed the total available bandwidth of the $\mathrm{SC}$, denoted by $B W_{S C}$, in order to guarantee service to all users. This condition is expressed as:

$$
\sum_{i \in I} \Lambda_{i}(T)=\Lambda_{A}(T)+\Lambda_{B}(T)=b \cdot B W_{S C}, b \in[0,1] .
$$

In the following sections, we introduce a model for the calculation of the total cost of the SC, taking into account the energy consumption due to the traffic load, and we study different techniques that share this cost among the operators according to their traffic patters.

\section{Cost And Energy Consumption Models}

In this section, we, first, introduce a model to estimate the total cost of a SC deployment and, then, we describe a model for the SC energy consumption.

\section{A. Cost Analysis Breakdown}

The cost of a SC consists of the CapEx, for deployment and installation, and the OpEx for its operation. Chen et al. presented a detailed breakdown of the CapEx and OpEx for macrocells in [14]. Following a similar approach and taking into account the typical cost of SC equipment [15], we have adapted the model in order to estimate the different costs in the case of a SC. 
Table I shows the CapEx and OpEx breakdown for SCs, whereas the respective values for the BS case ([14]) are also given for reference. In particular, the CapEx consists of four factors: i) the cost $C_{S C}^{C}$ for the small cell equipment ii) the cost for deployment and installation of the $\mathrm{SC}$, denoted by $C_{\text {Site }}^{C}$, iii) the cost $C_{R N C}^{C}$ of the Radio Network Controller (RNC), and iv) the cost for backhaul transmission equipment $\left(C_{B T}^{C}\right)$ that is almost negligible in the case of SCs. The OpEx, on the other hand, can be broken down to four terms: i) the cost of backhaul transmission $\left(C_{B T}^{O}\right)$, corresponding to the bandwidth needed to serve the traffic and calculated according to a simple leased line pricing, ii) the cost $C_{\text {Site }}^{O}$ for site leasing, iii) the cost $C_{O M}^{O}$ for operation and maintenance, and iv) the electric power cost $C_{P w}^{O}$ that depends on the power consumption to serve the traffic $\lambda(t)$ of the SC.

TABLE I: CapEx and OpEx breakdown for BSs and SCs

\begin{tabular}{c|c|c|c}
\hline CapEx & $C_{C a}$ & $\mathrm{BS}$ & $\mathrm{SC}$ \\
\hline BS/SC equipment & $C_{B S}^{C}, C_{S C}{ }_{S}$ & $c_{0}$ & $c_{0} / 70$ \\
Site installation and buildout & $C_{S i t e}^{C}$ & $c_{0} / 4$ & $c_{0} / 20$ \\
RNC equipment & $C_{R N C}^{C}$ & $3 c_{0} / 2$ & $c_{0} / 210$ \\
Backhaul transmission equipment & $C_{B T}^{C}$ & $c_{0} / 4$ & 0 \\
\hline \hline OpEx & $C_{O p}$ & & \\
\hline Backhaul and transmission lease & $C_{B T}^{O}$ & $c_{0} / 4$ & $c_{0} / 20$ \\
Site lease & $C_{S i t e}^{O}$ & $c_{0} / 4$ & 0 \\
Operation \& Maintenance & $C_{O M}^{O}$ & $c_{0} / 16$ & $9 c_{0} / 700$ \\
Electric power & $C_{P w}^{O}$ & $c_{1} E_{B S}$ & $c_{1} E_{S C}$ \\
\hline
\end{tabular}

In order to calculate a realistic annual SC cost, we assume that the CapEx estimated from Table I is equally distributed within 5 years. Therefore, the annual cost of a SC, $C_{S C}(\lambda(t))$ is the sum of the CapEx that corresponds to one year and the traffic-dependent $\mathrm{OpEx}{ }^{2}$ :

$$
C_{S C}(\lambda(t))=C_{C a} / 5+C_{O p}(\lambda(t)) .
$$

As shown in Table I, the first seven cost values have been expressed with respect to the equipment cost of a macro BS $c_{0}$ measured in $€[14]$. The last term, i.e., the electric power, depends on the electricity charge per energy unit $c_{1}$, measured in $€ / \mathrm{kWh}$, and the energy consumption of a SC $E_{S C}(\lambda(t))$, which will be calculated in the next section. With these relations in mind, Eq.(4) is expressed as:

$$
C_{S C}(\lambda(t))=0.0768 \cdot c_{0}+c_{1} \cdot E_{S C}(\lambda(t)) .
$$

\section{B. Energy Consumption Analysis Breakdown}

Unlike macro BSs, the SC power consumption is loaddependent. The consumed energy $E_{S C}(\lambda(t))$ over a specific time interval $t \in[0, T]$ can be calculated as:

$$
E_{S C}(\lambda(t))=\int_{0}^{T} P_{S C}(\lambda(t)) \mathrm{d} t
$$

The relationship between the power consumption $P_{S C}(\lambda(t))$ of the SC and the relative transmission power $\left(P_{\text {out }}(\lambda(t))\right)$ can be formulated as a linear function. To that end, a linear approximation of the power model is adopted, given by [16]:

$$
P_{S C}(\lambda(t))=P_{0}+\Delta_{p} \cdot P_{\text {out }}(\lambda(t)), \quad 0<P_{\text {out }} \leq P_{\max }
$$

\footnotetext{
${ }^{2}$ To calculate the annual cost, the SC traffic $\left.\lambda(t)\right)$ must be averaged over a year.
}

where $P_{0}$ is the minimum power consumption in case of no traffic in the system and $\Delta_{p}$ is the slope of the load-dependent power consumption.

\section{Cost Sharing Policies AND Discrepancies}

In this section, we present four different policies for sharing the cost of a SC owned by a third party between two operators [17], [18] and we try to quantify the discrepancies between these methods. In addition, we propose a novel cost sharing policy, called Hybrid-Sharing, that combines two different strategies in order to provide a fairer cost distribution according to the particular traits of the SCs.

\section{A. Cost Sharing Policies}

Traffic-Volume (TV): According to TV, the cost of the SC is shared proportionally to the traffic volumes of the operators. Hence, the cost of operator $i$ is:

$$
C_{i}=\frac{\Lambda_{i}}{\sum_{j \in I} \Lambda_{j}} \cdot C_{S C}\left(\sum_{j \in I} \Lambda_{j}\right)
$$

where $\Lambda_{i}$ is the total traffic of operator $i \in\{A, B\}$ (Eq.(1)) and $C_{S C}\left(\sum_{j \in I} \Lambda_{j}\right)$ the total cost of the SC (Eq.(5)) when serving the aggregate traffic of all operators in $I^{3}$.

Operator-Peak (OP): According to OP, the SC cost is shared based on the traffic peak of the $i$ th operator within a time period $t \in T$, with respect to the sum of the individual traffic peaks of all operators sharing the SC. Thus, the cost of operator $i$ is:

$$
C_{i}=\frac{\max _{t \in T} \lambda_{i}(t)}{\sum_{j \in I} \max _{t \in T} \lambda_{j}(t)} \cdot C_{S C}\left(\sum_{j \in I} \Lambda_{j}\right) .
$$

Aggregate-Peak (AP): In AP, the cost is shared according to the traffic of the $i$ th operator with respect to the traffic peak of the aggregated SC traffic, which occurs at a particular time period $t_{m}$. Consequently, the cost of operator $i$ is:

$$
C_{i}=\frac{\lambda_{i}\left(t_{m}\right)}{\sum_{j \in I} \lambda_{j}\left(t_{m}\right)} \cdot C_{S C}\left(\sum_{j \in I} \Lambda_{j}\right)
$$

Shapley-Value (SV). Shapley-value is a solution concept in cooperative game theory employed to predict a unique expected payoff allocation when different coalitions may be formed among the players. To understand the concept behind the SV calculation, consider the following example. If a SC is leased by operator A alone, then A must assume the total CapEx of the SC, as well as the OpEx that corresponds to its traffic. If $\mathrm{B}$ decides to join the $\mathrm{SC}$ after $\mathrm{A}$, then $\mathrm{B}$ must only pay the OpEx corresponding to its traffic (i.e., its marginal contribution), given that $\mathrm{A}$ has already covered the remaining $\mathrm{SC}$ expenses. Accordingly, the marginal contribution of operator A would be different if the arrival order of the two operators in the coalition was changed.

\footnotetext{
${ }^{3}$ Note that for convenience, all time notations have been dropped, when not necessary.
} 
In our specific case, we have $S \subset I$ possible coalitions of the two operators. The set of coalitions is $S=$ $\{\{A\},\{B\},\{A, B\}\}$, where $\{A\}$ and $\{B\}$ are the cases when each operator owns its own SC and $\{A, B\}$ is the grand coalition where the two operators share an SC. The cost of each coalition $C(S)$ is given by Eq.(5), by considering the traffic of operator $A, B$ and the aggregate traffic of both, respectively. The SV shares the total cost of the SC to the operators in a way proportional to each operator's average marginal contribution, after considering all the possible permutations $\Pi$ of the operators' arrival order to the grand coalition. If $\pi \in \Pi$ any permutation of operators, we define $S(\pi, i)$ as the set of operators that arrived to the coalition before operator $i$. Then, the Shapley value of the $i$ th operator, denoted by $\phi_{i}(C)$, is given by:

$$
\phi_{i}(C)=\frac{1}{|I| !} \cdot \sum_{\pi \in \Pi}(C(S(\pi, i))-C(S(\pi, i) \backslash i)),
$$

and the cost of operator $i$ is:

$$
C_{i}=\frac{\phi_{i}(C)}{\sum_{j \in I} \phi_{j}(C)} \cdot C_{S C}\left(\sum_{j \in I} \Lambda_{j}\right)
$$

The SV is considered the most fair solution for cost sharing but requires high complexity in terms of computational power (to calculate the marginal costs in all possible coalitions). To achieve a compromise between complexity and fairness, we propose a hybrid cost sharing solution, described next.

Hybrid-Sharing (HS): Let us emphasize that telecommunication operators design their networks based on the peak traffic utilization, even though the network remains underutilized during a large part of the day. As a result, the peak traffic also determines the backhaul equipment that should be employed. Therefore, the AP policy seems to be the most appropriate technique to share the operational cost of the backhaul $\left(C_{B T}^{O}\right)$. On the other hand, the TV method is a simple and fair way to share the costs that directly depend on the traffic load. Hence, we propose a hybrid policy (HS) that combines these two methods and calculates the cost of operator $i$ as:

$$
C_{i}=\frac{\lambda_{i}\left(t_{m}\right)}{\sum_{j \in I} \lambda_{j}\left(t_{m}\right)} \cdot C_{B T}^{O}+\frac{\Lambda_{i}}{\sum_{j \in I} \Lambda_{j}} \cdot\left(C_{C a}+C_{S i t e}^{O}+C_{O M}^{O}+C_{P w}^{O}\right) .
$$

\section{B. Cost Sharing Policies Discrepancies}

Interesting discrepancies are observed among the aforementioned cost sharing policies, since each of them results in different pricing schemes for the operators. Hence, we introduce the discrepancy metric, which quantifies the difference in the operator's costs when two distinct cost sharing policies are employed. To that end, the discrepancy of the two policies is defined as:

$$
d\left(C_{i}^{1}, C_{i}^{2}\right)=\frac{C_{i}^{1}-C_{i}^{2}}{C_{i}^{1}} \cdot 100 \%,
$$

where $C_{i}^{1}$ and $C_{i}^{2}$ denote the costs of operator $i \in I$ according to the policy 1 and 2 , respectively.

\section{PERFormance EVAluation}

In this section, we present the scenarios under study along with the analytical results of the different cost sharing policies.

\section{A. Scenario}

We consider the scenario depicted in Fig. 1, where two operators $(A$ and $B$ ) lease the capacity of a third party SC. To assess the performance of the cost sharing algorithms (TV, OP, AP, SV and HS), we consider the four traffic patterns shown in Fig. 2 with varying values of the traffic load volume (i.e., various values for the parameter a). Table II summarizes the key parameters employed in the cost and energy breakdown analysis explained in Section IV. Regarding the discrepancies, $\mathrm{SV}$ is used as the reference policy ${ }^{4}$ and the most extreme percentage differences of the other policies with regard to the $\mathrm{SV}$ are highlighted in each case.

TABLE II: System Parameters

\begin{tabular}{c|c||c|c}
\hline Parameter & Value & Parameter & Value \\
\hline Bandwidth & $30 \mathrm{Mb} / \mathrm{s}$ & $P_{\max }$ & $24 \mathrm{dBm}$ \\
$P_{0}$ & $4.76 \mathrm{~W}$ & $\Delta_{p}$ & 16.15 \\
$\mathrm{a}, b$ & {$[0,1]$} & $c_{0}, c_{1}$ & $20000 €, 0.1 €$ \\
\hline
\end{tabular}

\section{B. Performance Results}

We begin the performance evaluation by emphasizing the energy efficiency gains that can be achieved through infrastructure sharing. Even though these gains are independent of the adopted cost sharing scheme, they can strongly motivate the operators to cooperate, thus creating the need to select effective cost policies. Figure 3 depicts the energy efficiency gains achieved through infrastructure sharing versus the parameter $b$ that determines the SC occupation. More specifically, we examine two scenarios: i) a scenario where the SC is shared among two operators (Sharing), and ii) a baseline scenario where each operator owns its own SC (NoS) and, hence, no cooperation takes place. As we can see, for low SC utilization (i.e., $b=0.1$ ), the energy efficiency in shared networks is significantly higher, as we avoid the deployment of numerous SCs. As the traffic load increases, the percentage of the gain decreases, but there is no cross point, since sharing is more energy efficient in all cases. Motivated by these results, in the remainder of the paper, we study various ways and policies to share the SC cost among operators, focusing on the applicability, the complexity and the fairness of the different solutions.

Figure 4 illustrates the costs of the operators $\mathrm{A}$ and $\mathrm{B}$ considering the traffic patterns of Fig. 2a (Case I), where the traffic patterns follow a typical distribution and their peaks coincide in time. In this figure, we observe that the discrepancies of the different policies are more intense when the the difference in the traffic load of the two operators is high (i.e., $\mathrm{a}=0.1$ ). As the difference in their traffic load decreases, the discrepancies fade and, eventually, all policies provide the same outcome for equal traffic volume (i.e., $\mathrm{a}=1$ ). It is also worth noticing that, in this scenario, OP and AP cannot be distinguished, as the operator and the aggregated peak traffic takes place at the same time. Regarding our proposed policy, we can see that HS provides costs between TV and OP (or AP), as it combines the properties of both techniques.

\footnotetext{
${ }^{4}$ Despite its complexity, $\mathrm{SV}$ is assumed as the most fair solution for cost sharing.
} 


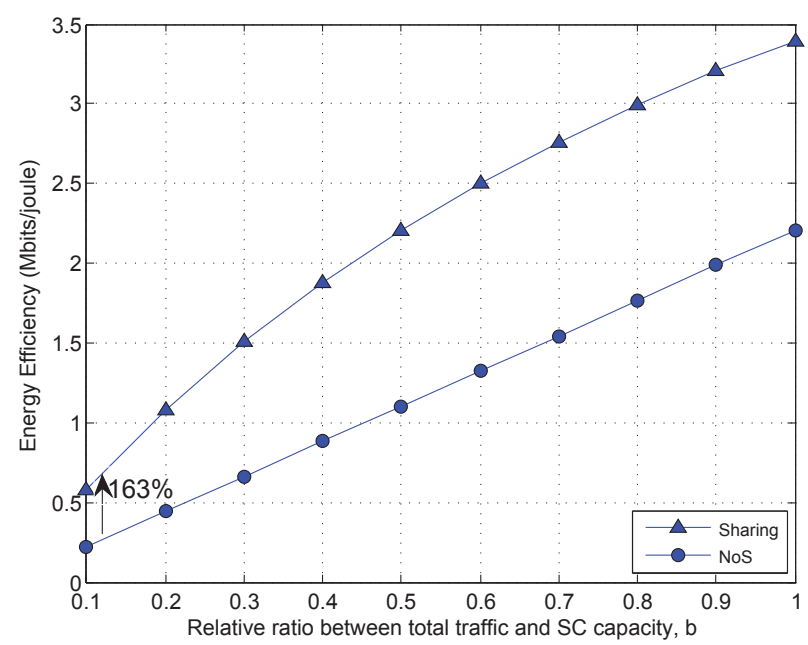

Fig. 3: Total Network Energy Efficiency

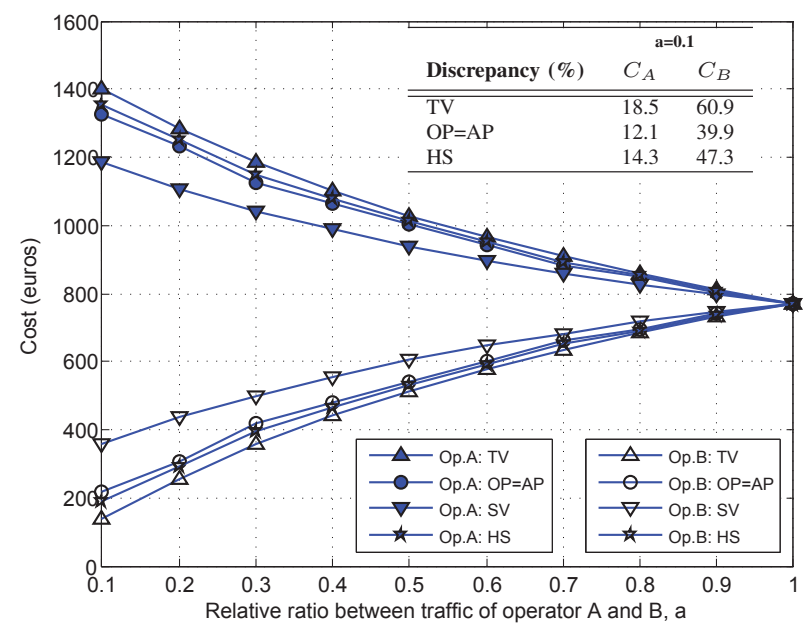

Fig. 4: Costs of operators given the traffic pattern of Case I (Fig. 2a) and cost sharing policies discrepancies in [\%]

Figure 5 presents the costs for the two operators according to the different policies, considering a scenario where the traffic follows again a typical distribution, but the peaks take place at different times during the day (Fig. 2b). Unlike the previous case, in this figure, OP and AP sharing policies have distinct performance, since the aggregated peak utilization time does not coincide with the maximum traffic value of each individual operator. In particular, although OP still provides similar outcome with the TV policy, the discrepancy between $\mathrm{AP}$ and SV is extremely higher. However, similar to the previous case, the proposed HS provides an intermediate solution by considering different parts of the cost using different techniques.

In Fig. 6 and 7, we study the operators cost in case of bursty traffic models (Fig. 2c and 2d, respectively). Unlike the previous cases, here we consider that the parameter a affects only the high traffic period. For example, when $\mathrm{a}=1$, the peak traffic of operator B would be higher than operator's A peak traffic, since i) both operators are expected to have the

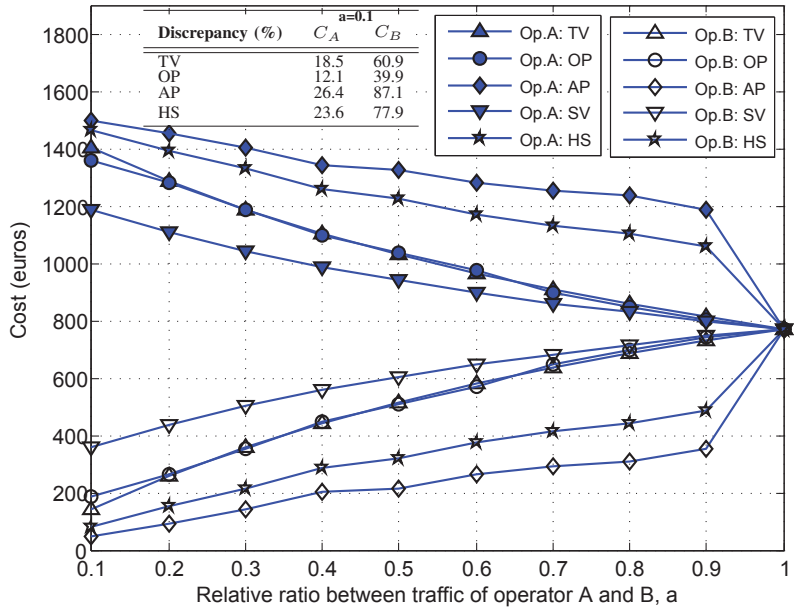

Fig. 5: Costs of operators given the traffic pattern of Case II (Fig. 2b) and cost sharing policies discrepancies in [\%]

same traffic volume and ii) during the rest of the day, operator A has slightly higher traffic compared to operator B.

In particular, in Fig. 6, we notice again that the OP and the AP policy have the same outcome, as the peak values coincide in time, despite their different values. On the other hand, TV and SV exhibit similar performance, converging to the same point when the traffic volume of the operators is the same (i.e., $\mathrm{a}=1$ ). Our proposed solution (HS) manages to reduce the great discrepancy of OP and AP, without neglecting the impact of the peak traffic in the system. In Fig. 7, we can see that $\mathrm{OP}$ and AP have similar behavior, although the peak times take place in different time periods during the day. However, HS achieves again lower discrepancies from both strategies, with regard to the reference policy (SV).

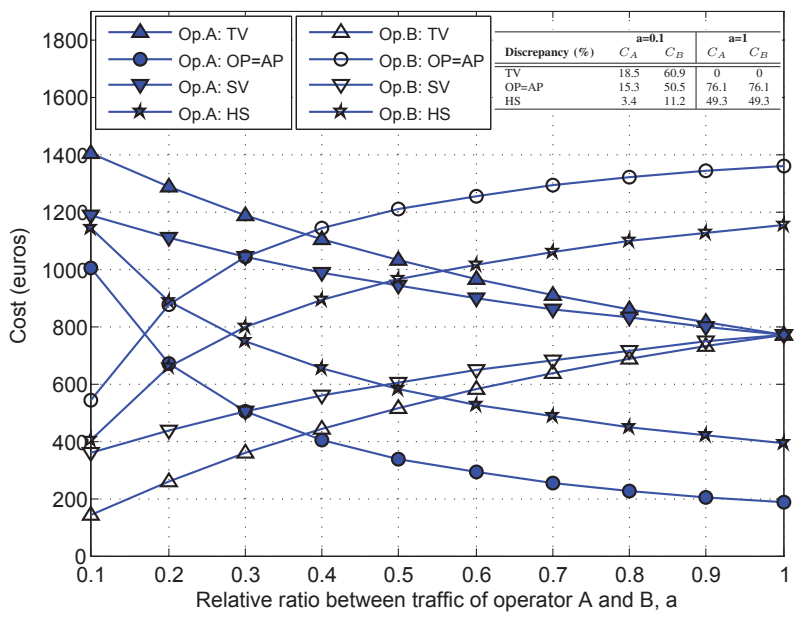

Fig. 6: Costs of operators given the traffic pattern of Case III (Fig. 2c) and cost sharing policies discrepancies in [\%]

\section{Discussion}

Based on the analysis in Section IV-A and the analytical results, we have shown that the proposed HS policy considers 


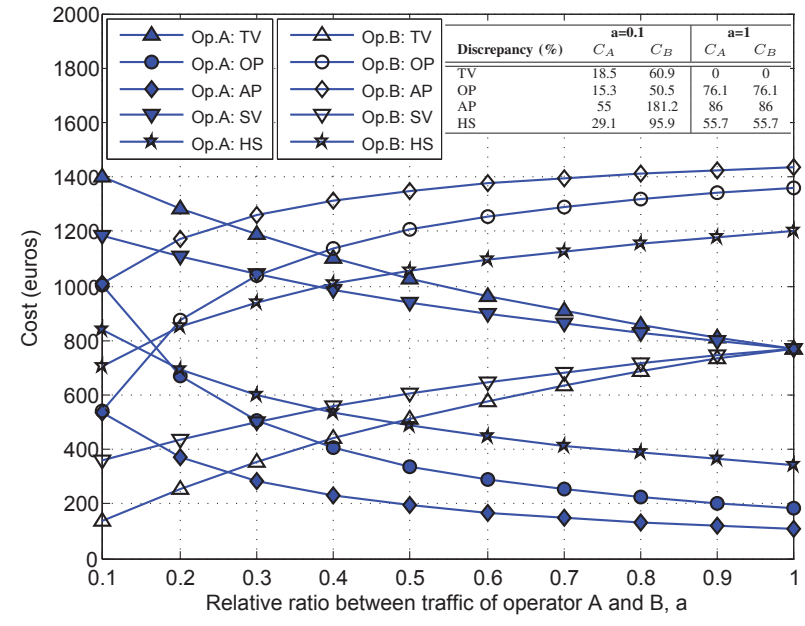

Fig. 7: Costs of operators given the traffic pattern of Case IV (Fig. 2d) and cost sharing policies discrepancies in [\%]

different characteristics of the traffic pattern, unlike TV, OP and $\mathrm{AP}$, which examine only one aspect (either volume or peak times). In addition, through an extensive assessment, we have identified significant discrepancies between the sharing policies and the SV policy, which was used as a reference strategy. In particular, the TV method shows a steady performance regardless of the variations in the traffic profile (i.e., its discrepancy remains stable in all cases). Hence, despite its simplicity, the TV does not seem effective for future networks, where the different traffic patterns are expected to play a crucial role. On the other hand, the OP and the AP policies are greatly affected by the adopted traffic pattern, especially in bursty traffic conditions, due to the misalignment of the operators' peak values.

However, in future wireless networks, where third parties are expected to offer services through SCs, a cost sharing policy that considers the individual and specific needs of the operators is more than necessary. In particular, different traffic patterns affect the shared costs and, as a result, different factors of traffic, such as volume, peak times and burstiness, should be taken into account. To that end, our proposed approach (HS) estimates a cost according to both the traffic volume and the peak time traffic, offering a fairer solution to the operators. Therefore, the discrepancies of the proposed HS policy fluctuate between the discrepancies of the other policies, independently of the particular scenario. It is worth noting that HS policy is less complex than SV, as it does not require extra overhead information, thus being a useful tool for sharing the network cost among the operators in the near future.

\section{CONClusion}

The possibility of a third party that provides a tier of SCs in HetNets can provide significant gains in energy efficiency, but at the same time raises important issues with regard to the SC cost sharing among different operators. In this paper, we introduced an accurate cost model for the SCs that takes into account the traffic load for a precise estimation of the energy consumption. In order to effectively share this cost, we investigated four different state-of-the-art cost sharing techniques and we introduced a new hybrid policy that achieves a trafficaware sharing of the total expenses. Our results highlighted the potential energy efficiency gains in the network, along with the fair sharing of the SC cost that can be achieved through our proposed policy. In our future work, we are planning to exploit these results by providing the operators with the necessary incentives to share the SC infrastructure during low traffic periods in order to be able to switch off the macro BSs, thus reducing further the energy consumption inside the network.

\section{ACKNOWLEDGMENT}

This work has been funded by the Research Projects GREENET (PITN-GA-2010-264759), CO2GREEN (TEC2010-20823), GREEN-T (TSI-020400-2011-16-CP8006) and GEOCOM (TEC2011-27723-C02-01).

\section{REFERENCES}

[1] Cisco Whitepaper, Cisco Visual Networking Index: Global Mobile Data Traffic Forecast Update, 2012-2017, February 2013.

[2] A. Ghosh, N. Mangalvedhe, R. Ratasuk, B. Mondal, M. Cudak, E. Visotsky, T.A. Thomas, J.G. Andrews, P. Xia, H.S. Jo, H.S. Dhillon, and T.D. Novlan, "Heterogeneous cellular networks: From theory to practice," in IEEE Communications Magazine, vol. 50, no. 6, pp. 54-64, June 2012.

[3] J.G. Andrews, "Seven ways that HetNets are a cellular paradigm shift," in IEEE Communications Magazine, vol. 51, no. 3, pp. 136-144, March 2013.

[4] Mobile Network Sharing report 2010-2015, Development, Analysis \& Forecasts, Market Study, Visiongain, 2010.

[5] D. E. Meddour, T. Rasheed, and Y. Gourhant, "On the role of infrastructure sharing for mobile network operators in emerging markets," in Elsevier Computer Networks, vol. 55, no. 7, pp. 1567-1591, May 2011.

[6] A. Bousia, E. Kartsakli, A. Antonopoulos, L. Alonso, and C. Verikoukis, "Game Theoretic Approach for Switching Off Base Stations in MultiOperator Environments," in Proceedings of the IEEE International Conference on Communications (ICC), 2013.

[7] C. Beckman and G. Smith, "Shared networks: making wireless communication affordable," in IEEE Wireless Communications, vol. 12, pp. 78-85, April 2005.

[8] J. Markendahl and A. Ghanbari, "Shared small cell networks multioperator or third party solutions - or both?," in 11th International Symposium on Modeling Optimization in Mobile, Ad Hoc Wireless Networks (WiOpt), 2013.

[9] S. Paris, F. Martignon, I. Filippini, and A. Capone, "A truthful auction for access point selection in heterogeneous mobile networks" in Proceeding of the IEEE International Conference on Communications (ICC), 2012.

[10] K. Zhu, E. Hossain, and D. Niyato, "Pricing, spectrum sharing, and service selection in two-tier small cell networks: A hierarchical dynamic game approach," IEEE Transactions on Mobile Computing, vol. 99, no.PrePrints, pp. 1, 2013.

[11] L. Gao, G. Iosifidis, J. Huang, and L. Tassiulas, "Economics of Mobile Data Ofoading," in Proceedings of the 29th IEEE SDP Workshop, 2013.

[12] L. Gyarmati, M. Sirivianos, N. Laoutaris, "Sharing the Cost of Backbone Networks: Simplicity vs. Precision," in Proceedings of the IEEE Conference on Computer Communications Workshops (INFOCOM), 2012.

[13] L. Gyarmati, R. Stanojevic, M. Sirivianos, and N. Laoutaris, "Sharing the Cost of Backbone Networks: Cui Bono?," in Proceedings of the ACM Conference on Internet Measurement (IMC), 2012.

[14] Y. Chen, S. Zhang, and S. Xu, "Characterizing Energy Efficiency and Deployment Efficiency Relations for Green Architecture Design," in Proceedings of the IEEE International Conference on Communications (ICC), 2010.

[15] www.smallcellforum.org

[16] G. Auer, V. Giannini, C. Desset, I. Gdor, P. Skillermark, M. Olsson, M.A. Imran, D. Sabella, M.J. Gonzalez, O. Blume, and A. Fehske, "How Much Energy Is Needed To Run A Wireless Network?," in IEEE Wireless Communications, vol.18, no.5, pp.40-49, October 2011.

[17] A. Qureshi, R. Weber, H. Balakrishnan, J. Guttag, and B. Maggs, "Cutting the electric bill for internet-scale systems," in Proceedings of the ACM Conference on Data Communication (SIGCOMM), 2009.

[18] L. S. Shapley, "A value for n-person games," in Annals of Mathematical Studies, 1953. 